COMMENT. Rasmussen's encephalitis is characterized by intractable seizures of focal onset, usually an epilepsia partialis continua, and chronic inflammation with progressive hemispheric neurologic deterioration. In the final residual, atrophic stage of the disorder, seizures decrease in frequency and the neurologic deficit becomes static. The differentiation between a viral cause or an autoimmune mechanism remains to be determined.

\title{
PROSPECTIVE DIAGNOSIS AND TREATMENT OF PANDAS
}

Twelve school-aged children with new-onset pediatric autoimmune neuropsychiatric disorder with group A streptococcal infection (PANDAS) were identified over a 3-year period (1998-2000) at the University of Rochester, NY. An acute onset of severe obsessive-compulsive disorder was associated with an acute group-A beta-hemolytic streptococcal (GABHS) pharyngitis, confirmed by positive throat culture and/or rapid antigen detection, and in 3 cases, very high antideoxyribonuclease antibody titers. All patients were treated with penicillin, amoxicillin or a cephalosporin for 10 days, and eradication of GABHS was followed by immediate resolution of the OCD symptoms. Recurrence of OCD in 6 patients was associated with acute GABHS that responded to antibiotic therapy. The age at onset of OCD was 5-12 years, predominantly in boys (1.4:1), and the initial OCD episode occurred from September through April, the peak seasonal occurrence of GABHS. Symptoms of OCD, meeting DSMIV diagnostic criteria, included compulsive hand washing or excessive toilet hygiene, compulsive daytime urinary urgency without infection, and age-inappropriate separation anxiety when leaving their mothers. Four children (33\%) had neurological disorders in addition to OCD, including recurrent tics, transient $\mathrm{ADHD}$, or choreiform movements. This is the first prospective study to confirm the PANDAS syndrome and its response to treatment with penicillin. (Murphy ML, Pichichero ME. Prospective identification and treatment of children with pediatric autoimmune neuropsychiatric disorder associated with group A streptococcal; infection (PANDAS). Arch Pediatr Adolesc Med April 2002;156Ú356-361). (Reprints: Michael E Pichichero MD, Elmwood Pediatric Group, University of Rochester Medical Center, 601 Elmwood Avenue, Box 672, Rochester, NY).

COMMENT. This study corroborates the association between streptococcal infection and the acute onset of neuropsychiatric symptoms. It also demonstrates the benefit of treatment with penicillin for the tonsillitis or pharyngitis with concomitant recovery from the OCD, tics and sometimes ADHD symptomatology. A possible diagnosis of PANDAS should be considered in new cases of OCD or tics, especially when symptoms are acute in onset.

\section{SEIZURE DISORDERS}

\section{SCN1A GENE MUTATIONS IN SEVERE INFANTILE MYOCLONIC EPILEPSY}

Ten novel mutations of SCN1A were found in in a pair of monozygotic twins and 12 unrelated Japanese infants with severe myoclonic epilepsy in infancy (SMEI) examined at the Brain Science Institute, Saitama; and National Epilepsy Center, Shizuoko, Japan. Of the 10 heterozygous mutations, 3 were frameshift and 7 nonsense mutations. These rather than missense mutations are the major causes of SMEI. Missense mutations of the same gene result in generalized epilepsy with febrile sezures plus (GEFS plus). (Sugawara T, Mazaki-Miyazaki E, Fukushima K et al. Neurology April (1 of 2) 2002;58:1122-1124). (Reprints: Dr K Yamakawa, Laboratory 
for Neurogenetics, RIKEN Brain Research Institute, Hirosawa 2-1, Wako-shi, Saitama 3510198, Japan).

CPMMENT. The syndrome of severe myoclonic epilepsy in infancy is characterized by normal development initially, onset of seizures during the first year, beginning with generalized or unilateral febrile clonic seizures, and followed by the secondary appearance of myoclonic seizures and partial seizures that are intractable and complicated by ataxia and mental deterioration. Genetic predisposition suggested by family history and concordant disease in monozygotic twins appears to be based on frameshift and nonsense mutations in the SCN1A gene.

\section{HISTORY OF WEST SYNDROME}

Historical details about Dr West, his son, James Edward West, the patient with infantile spasms, and the coining of the eponym 'West syndrome' are researched by pediatric neurologists and colleagues at the University of Nijmegen, the Netherlands; and University of California at Irvine. WJ West, a general practitioner at Tonbridge, UK, wrote a letter to the Lancet in 1841 (1:724725), entitled 'On a peculiar form of infantile convulsions.' To paraphrase, the infant was well until 4 months of age, when he began slight bobbings of the head forward, later a complete heaving of the head forward toward his knees, and then immediately relaxing into the upright position, similar to attacks of emprosthotonus. The patient was born Feb 13, 1840 and died at 20 years of age on Sept 27, 1860, having spent the last 7 years of his life in an Asylum for the FeebleMinded in Redhill, UK. He is buried with his father in the cemetery of the St Peter and Paul parochial church in Tonbridge. When Dr West's treatments failed (leeches, calomel, phlogiston, hot baths, alterantia drugs, opium), he consulted Drs Clarke and Locock in London, who called the seizures by the term 'Salaam convulsions,' having seen two similar cases previously. Subsequent early publications include those of Newnham (1849), using the term 'eclampsia nutans,' Fere (1883) who described 'tic de Salaam or salutation, and Zellweger (1948), who, like Fere, distinguished between symptomatic and idiopathic infantile spasms. Gibbs and Gibbs (1952) introduced the term 'hypsarrhythmia' and described the classical triad of diagnostic criteria, 'spasms, hypsarrhythmia, and mental retardation.' The term West syndrome was first suggested by Gastaut (1960) at the 9th Colloque de Marseille on infantile spasms, then called 'infantile myoclonic encephalopathy with hypsarrhythmia' (IMEH). (Eling P, Renier WO, Pomper J, Baram TZ. The mystery of the Doctor's son, or the riddle of West syndrome. Neurology March (2 of 2);58:953-955). (Reprints: Dr Paul Eling, NICI, PO Box 9104, 6500 HE Nijmegen, the Netherlands).

COMMENT. The advances made in the riddle of West syndrome, since it was first described 160 year ago, are the descriptive electroencephalographic abnormalities (hypsarrhythmia) by the Gibbs' (1952) and the introduction of ACTH (1958) by Sorel and Dusaucy-Bauloye, a largely empiric therapy. A study of etiologic factors in 61 patients with infantile West syndrome was published in 1962 (Millichap, Bickford, Klass, Backus, in Epilepsia 3:188), but the pathophysiology of the syndrome still eludes us (Baram, 1993). Incidentally, Tonbridge, the town in UK where West and his son are buried, is situated about 14 $\mathrm{km}$ north of Tunbridge Wells, the site of the Spastics Society and editorial office of the journal 'Dev Med Child Neurol.' 